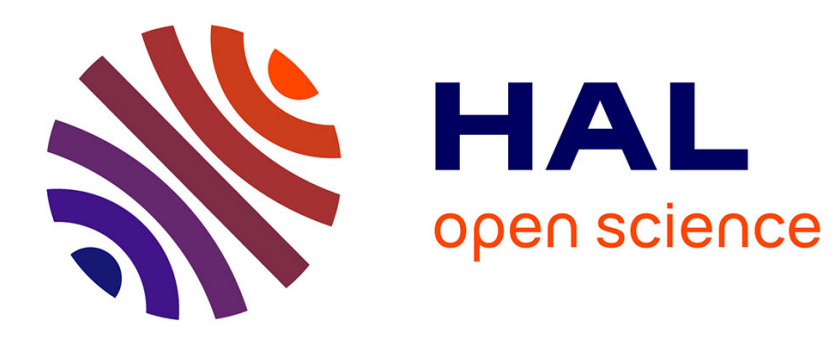

\title{
Le singulier selon Gustave Guillaume
}

Philippe Monneret

\section{To cite this version:}

Philippe Monneret. Le singulier selon Gustave Guillaume. L'information grammaticale, 2010, 126, pp.51-57. hal-01037908

\section{HAL Id: hal-01037908 https://hal.science/hal-01037908}

Submitted on 23 Jul 2014

HAL is a multi-disciplinary open access archive for the deposit and dissemination of scientific research documents, whether they are published or not. The documents may come from teaching and research institutions in France or abroad, or from public or private research centers.
L'archive ouverte pluridisciplinaire HAL, est destinée au dépôt et à la diffusion de documents scientifiques de niveau recherche, publiés ou non, émanant des établissements d'enseignement et de recherche français ou étrangers, des laboratoires publics ou privés. 


\title{
LE SINGULIER SELON GUSTAVE GUILLAUME
}

\section{Philippe MONNERET}

Parmi les tâches qui incombent aux linguistes s'inscrivant dans la mouvance guillaumienne, la clarification de la terminologie demeure une préoccupation essentielle. Bien que le Dictionnaire terminologique de la systématique du langage (Boone et Joly, 1996) ait marqué une avancée significative à cet égard, le foisonnement du métalangage guillaumien, son évolution ou ses variations au cours du temps, font que de nombreuses questions restent en suspens, non seulement pour qui s'intéresse, en historien, à la genèse de la théorie guillaumienne, mais aussi et surtout pour qui considère que la systématique du langage, nullement réductible à un objet d'histoire, reste une théorie féconde et exploitable pour les linguistes contemporains. Le caractère plus ou moins nécessaire de ce genre de clarification terminologique dépend bien entendu des questions de l'on se pose, ou qui émergent à une époque donnée et c'est sans doute pourquoi certains flottements demeurent invisibles tant que personne n'a éprouvé de gêne à leur endroit. Ainsi, il semble que les lecteurs de Guillaume n'ont guère été embarrassés, jusqu'à présent, par une variation terminologique qui touche pourtant un point crucial de la théorie guillaumienne, sa marque de fabrique la plus notoire : la tension " universel / singulier », qui est explicitement thématisée dès Temps et verbe (Guillaume, 1965 [1929]), et sera symbolisée à partir des années cinquante par le fameux «tenseur binaire ». Cette variation, perçue comme insignifiante, consiste en une alternance du couple «universel/singulier» avec le couple « général / particulier », qui conduit à s'interroger sur le sens des notions de singularité ou de particularité comme sur celui des notions d'universalité et de généralité. Le caractère insignifiant d'une telle variation apparaît bien dans l'article «généralisation/particularisation » du Dictionnaire terminologique de la systématique du langage :

\begin{abstract}
Ce sont «les deux mouvements de pensée les plus généraux, inhérents à l'esprit humain et inséparables de son activité » (Guillaume, 1943-44 : 19). Ces mouvements fondamentaux opèrent entre les pôles que sont l'universel (général) et le singulier (particulier). Généralisation et particularisation sont les deux opérations souveraines de la pensée humaine qui tiennent dans leur dépendance toutes les autres. (Boone et Joly, $1996:$ 199)
\end{abstract}

La question qui se pose alors n'est pas tant de savoir si les oppositions « universel / singulier » et «général / particulier » sont indifférenciées pour le linguiste Gustave Guillaume ${ }^{1}$, mais de déterminer le sens de chacun de ces termes pour en délimiter l'usage possible en systématique du langage, et plus généralement en linguistique, voire en philosophie du langage ${ }^{2}$. On s'attachera ici uniquement à la

\footnotetext{
1 Rappelons que traditionnellement (par exemple dans la Logique de Port-Royal), les philosophes distinguent: 1) le général ou l'universel (idée ou terme pouvant représenter plusieurs choses ; $p$. ex. homme, ville, cheval) ; 2) le singulier (idée ou terme qui ne représente qu'une seule chose; p. ex. Socrate) ; 3) le particulier (idée ou terme, qui ne s'étend qu'à une partie indéterminée de ce qu'il représente, de son étendue; p. ex. quelque triangle). La proposition est dite universelle, singulière ou particulière selon que son sujet est lui-même universel, singulier ou particulier.

${ }^{2}$ Le problème du singulier, du particulier ou de l'individu est l'une des grandes questions de la philosophie médiévale. Cette question, en relation avec celle des universaux, est liée à celle du statut de la réalité, ainsi que de la possibilité de la connaissance. Si pour un nominaliste médiéval comme Ockham
} 
distinction singulier/particulier, et plus précisément au sens du singulier dans le modèle guillaumien, sujet dont on verra qu'il couvre déjà une très large matière ${ }^{3}$.

\section{LE SINGULIER ET LE PARTICULIER DANS LE SYSTÈME DU MOT}

La tension «Universel / Singulier» ou «Général / Particulier» intervient d'une manière décisive dans plusieurs systèmes de la langue. Je concentrerai mon propos sur quatre d'entre eux : le système du mot, la théorie de la sémantèse, le système du nombre et le système de l'article.

\subsection{Le particulier dans les premiers états de la théorie du mot: le rôle de la subduction}

La théorie du mot se développe chez Guillaume à partir de 1938-1939, c'est-à-dire dès les premières Leçons. Dans l'article de 1938, «Théorie des auxiliaires et examen de faits connexes », l'opération productrice du mot est décrite de la manière suivante :

Le mot, dans les langues très évoluées qui nous sont familières, est le produit d'une double genèse : une genèse matérielle, qui en détermine l'être particulier (la signification), une genèse formelle qui en détermine l'être général (la partie du discours : substantif, verbe, adverbe, etc.) [...] Le mot s'évoque en nous sous une forme générale dont nous ne saurions nous passer. Il nous est tout à fait impossible d'évoquer un mot qui ne serait que mot, qui ne serait pas en même temps et de surcroît, substantif, verbe, etc. L'être général nous est ici aussi indispensable que l'être particulier. (Guillaume 1964b [1938] : 77)

L'enjeu de cette théorie du mot dans le traitement de la question des auxiliaires est lié à la théorie de la subduction ${ }^{4}$. En effet, les auxiliaires (p. ex. avoir dans avoir marché) sont analysés comme des mots dont la genèse matérielle, celle qui mène à l'être particulier de la signification, est interrompue avant son terme. L'auxiliaire est donc un mot complet dans sa forme (il est un verbe qui se conjugue à tous les modes et à tous les temps) mais incomplet dans sa matière, la matière manquante étant fournie par un autre mot (p. ex. le participe passé marché dans avoir marché). On remarquera en premier lieu que dans ce texte, Guillaume utilise exclusivement des termes «particulier » et «général ». Quant à la nature du particulier envisagé dans cette formulation de la théorie du mot, elle se dégage du contraste entre le verbe fortement atteint par le

par exemple, le singulier revêt une importance cruciale, c'est évidemment parce que le champ du singulier est pour lui co-extensif à celui de la réalité, ou de l'existence si l'on préfère : rien n'existe qui ne soit singulier (d'où le rapport avec le problème de la référence, que j'aborderai succinctement dans la dernière partie de cet article). Or, ces problématiques caractéristiques de la scolastique médiévale réapparaissent dans la philosophie contemporaine, sous des formes diverses : chez un Rosset par exemple, à qui l'on doit un livre original sur la question de la singularité (Rosset, 1979 ; repris dans Rosset, 2008), mais surtout, au-delà de ce cas isolé, dans toute la métaphysique analytique contemporaine, qui accorde une attention soutenue aux concepts d'universel, de singulier, de particulier ou évidemment d'individu. Dans le tableau des dix questions les plus disputées en métaphysique analytique proposé récemment par Frédéric Nef, plusieurs impliquent directement ou indirectement ces concepts : «Existe-t-il des essences individuelles ?», «L'identité est-elle nécessaire ? », «Peut-on se passer d'universaux ? », etc. (voir Nef, 2004 : 634). On notera en outre, à titre symptomatique, que la «métaphysique des particuliers » est aujourd'hui le nom d'un programme de recherche en philosophie (voir Nef, 2004 : 49).

${ }^{3}$ La distinction universel / général gagnerait bien sûr à être étudiée dans la même perspective, mais elle ne sera pas abordée dans le présent article.

${ }^{4}$ Sur la distinction entre subduction «ésotérique » et subduction « exotérique », voir (Guillaume 1964 [1938] : 74-75) 
processus de subduction, plus éloigné du particulier, et le verbe faiblement atteint par le processus de subduction, plus proche du particulier. Le particulier, comme terme d'un processus, est donc atteint lorsque la subduction est nulle ou, en d'autres termes, lorsque la genèse dite «matérielle» du mot est achevée, c'est-à-dire lorsque le signifié «matériel » du mot est clairement déterminable. Plus précisément, le particulier dont il est ici question s'applique à «l'être du mot» et Guillaume assimile ce qu'il appelle «l'être particulier du mot» à sa «signification», cet «être particulier du mot » étant opposé à un «être général du mot»qui n'est autre que la partie du discours. L'opposition du particulier au général ne correspond donc pas à deux états inversés d'un seul et même processus ${ }^{5}$ mais à deux processus nettement distincts du point de vue de leur nature : d'un côté la genèse d'un signifié «matériel », de l'autre celle d'un signifié « formel ». Si l'on considère le premier processus, le signifié «matériel » se caractérise par le fait qu'il peut se présenter comme «aisément fixable» (Guillaume 1964b [1938]: 75), lorsqu'il atteint le particulier (par exemple lorsque avoir signifie «posséder»), mais posséder un sens «moins pénétrable», "aussi facile à manier que difficile à fixer » (ibid.), dont on ne peut «concevoir clairement l'idée » (Guillaume 1964b [1938] : 77), lorsqu'il se situe à distance du particulier (par exemple lorsque avoir est utilisé comme auxiliaire). Le particulier renvoie donc ici à quelque chose comme une qualité sémantique maximale, qui se traduit par le fait que la signification du mot (au sens usuel de «signification») est aisément appréhendable. Une caractérisation assez vague, donc, que l'on pourrait compléter en considérant que la qualité sémantique de la signification matérielle d'un mot autorise une description, notamment lexicographique ${ }^{6}$. On notera enfin que le processus de genèse matérielle du mot, qui aboutit au particulier, est analysé d'une manière régressive : c'est à partir de la subduction que le contenu de la genèse matérielle du mot est présenté, et la subduction apparaît bien comme un processus régressif, au sens où elle est définie comme une dématérialisation effectuée à partir d'une matérialisation achevée et conclue au particulier.

\subsection{La confusion du particulier et du singulier dans la théorie du mot}

Guillaume revient sur le concept de subduction dans toutes les Leçons de décembre 1938, sans rien changer à l'analyse du concept lui-même mais en apportant quelques précisions intéressantes. Relativement à la question qui nous intéresse ici, une inflexion importante se produit dans la Leçon du 16 décembre, à l'occasion d'une réflexion sur le verbe comme partie du discours : le couple «Particulier / Général » est supplanté par le couple «Singulier / Universel ». C'est ce qui apparaît dans le schéma suivant (Guillaume, 1992 [1938] : 38) :

\footnotetext{
${ }^{5}$ Comme c'est le cas par exemple dans le système de l'article (voir infra).

${ }^{6}$ Voir par exemple l'article « Avoir » du Trésor de la Langue Française, qui est en mesure de fournir des synonymes pour la première série de sens («I. Emploi trans. Être en relation (concrète ou abstraite, permanente ou occasionnelle) avec quelqu'un ou quelque chose») où l'on trouve avoir au sens de «posséder» (et bien d'autres nuances sémantiques négligées par Guillaume), mais qui ne peut que se limiter à un classement morphosyntaxique pour les autres séries d'acceptions («II. Verbe copule de loc. verbales. »; « III. Emploi d'auxil. »; « IV. Verbe impers. Il y a. »).
} 


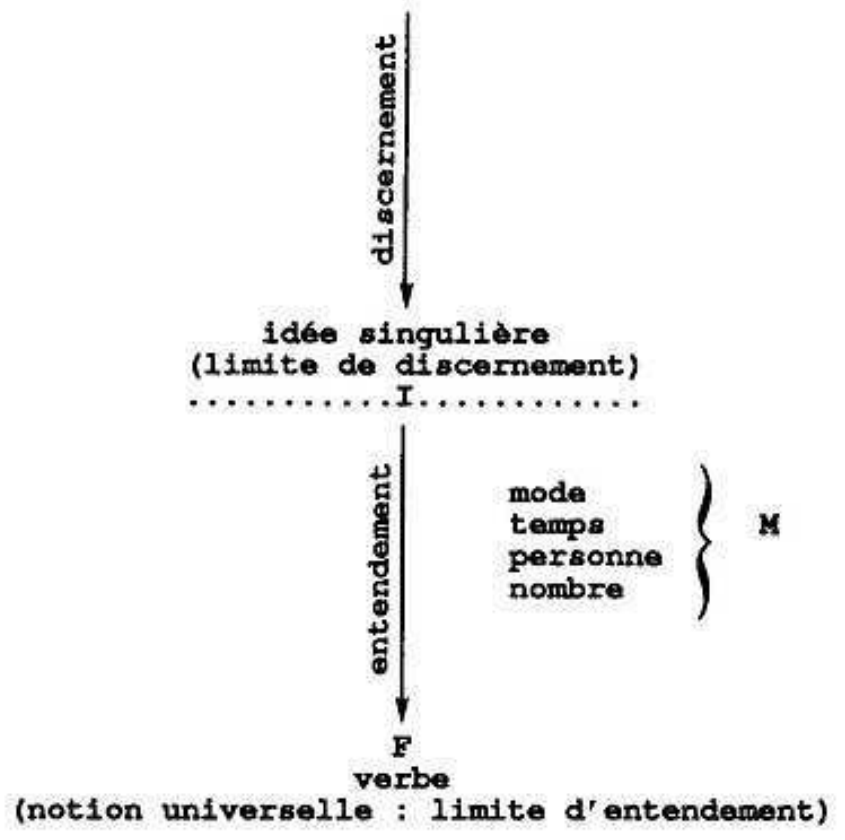

que Guillaume commente ainsi :

La genèse d'entendement conduit à la notion universelle de verbe, et entre le singulier (I) et l'universel (F) s'insèrent des indications médianes (M) de mode, de temps, de personne, de nombre. Cette insertion d'indications médianes est, qu'on le remarque bien, une nécessité de systématisation intellective. Le singulier, la notion discernée, marque le point de départ de l'universalisation d'entendement qui va faire du mot un verbe ; et l'universel, le point d'arrivée de l'entendement qui fait effectivement du mot un verbe. (Ibid.)

On ne trouve plus aucune trace dans cette Leçon (ni dans celles qui suivent au cours des années 1938-39) des termes «général» ni «particulier». Comment expliquer ce changement de terminologie, qui n'implique, je le rappelle, aucune modification au plan théorique ? L'hypothèse que je défendrai ici est la suivante : l'élimination du particulier au bénéfice du singulier provient de la prise en compte, par Guillaume, du singulier au sens numérique (par opposition au pluriel) et l'élimination du général au bénéfice de l'universel est une conséquence de cette introduction du mot «singulier », dès lors chargé d'une double signification : singulier vs pluriel ; singulier vs universel. C'est à la faveur d'une réflexion sur les applications possibles de la notion de subduction qu'apparaît la catégorie du nombre :

Il convient d'examiner à présent d'autres conséquences du procès de subduction. Il en est partout, et la genèse des morphèmes en particulier en procède, en quelque sorte, régulièrement. Je ne donnerai qu'un exemple, mais saisissant. Il existe en français le nombre un. Ce nombre, sous une forte subduction, a donné l'article, fait qui s'est produit aussi ailleurs. L'emploi d'une manière continuelle de l'article vise, nul n'en disconviendra, à tout autre chose que d'affirmer l'unité. La fonction de l'article est une fonction spéciale que j'étudierai au cours du second semestre. Ce que je veux indiquer seulement, c'est l'effet de la subduction qui a permis de passer de l'adjectif de nombre à l'article. (Guillaume, 1992 [1938] : 45)

Et Guillaume poursuit en opposant le nombre linguistique au nombre arithmétique :

À propos du nombre, il n'est peut-être pas sans intérêt de caractériser une distinction qui a son importance en linguistique générale. C'est celle du nombre spécifiquement linguistique et du 
nombre ambigu : linguistique et arithmétique. La série, historiquement identifiée, du nombre linguistique est : singulier, duel, triel, quatriel......pluriel ; en face, on a la série linguistiquearithmétique : un, deux, trois, quatre, etc.; et la série purement arithmétique : 1, 2, 3, 4, etc. (Ibid.)

Le singulier apparaît donc bien comme terme désignant un nombre linguistique - le premier élément d'une série «historiquement identifiée »-, alors qu'il signifiait au début de la même Leçon le terme d'un processus de genèse de la matière sémantique du mot. Nous reviendrons ultérieurement sur le rôle du singulier numérique à l'égard de la définition guillaumienne de la singularité et sur la relation entre le système du nombre et le système de l'article. Je me bornerai pour l'instant à relever en premier lieu que l'éviction du terme particulier au bénéfice du terme singulier n'engage ici que d'une manière minimale les notions de singularité et de particularité : le seul avantage du terme singulier est de permettre plus naturellement que le terme particulier l'intégration du singulier numérique. En aucune façon, ce changement n'est suscité par une réflexion sur la différence entre les concepts de singularité et de particularité.

On notera par ailleurs que le sens donné au concept de singularité (singularité de matière notionnelle) est précisé par les expressions «idée singulière », "notion discernée », qui confirment d'une part que Guillaume vise un singulier conceptuel ${ }^{7}$, et d'autre part que ce singulier s'oppose, en amont, ou si l'on préfère à un stade antérieur de la genèse d'entendement, non pas à un concept moins singulier (ou plus universel), mais à une notion indiscernée, non encore discernée, c'est-à-dire à un non-concept ${ }^{8}$.

Dans les états les plus tardifs de la théorie de la lexigenèse, le privilège du singulier au détriment du particulier semble se confirmer : les schémas qui présentent la théorie des aires glossogéniques ${ }^{9}$, dans laquelle la lexigénèse joue un rôle essentiel - puisque c'est à partir de la formation du mot que Guillaume distingue les trois aires (langues à caractères, langues à racines, langues à parties du discours) - sont très majoritairement fondés sur la polarité « Universel » / «Singulier ». Dans les Leçons de 1956-1957, ainsi que dans la première version de l'article «Observation et explication dans la science du langage » paru en 1958, les schémas de la lexigénèse présentent en effet régulièrement ces deux polarités. Toutefois, il n'est pas rare que la notion de particularisation réapparaisse dans le commentaire. Tel est le cas par exemple dans le texte suivant ${ }^{10}$ :

A l'opposé [des langues de l'aire prime], les langues dont nous avons l'accoutumance sont régies par la cinèse :

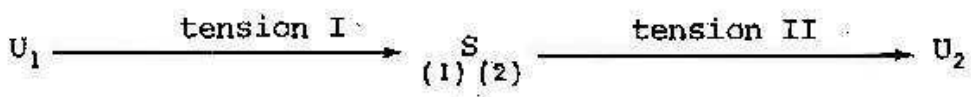

\footnotetext{
${ }^{7}$ Conceptuel plutôt que sémantique, puisque la matière notionnelle, au terme de la genèse d'entendement, n'est pas encore un mot. Ce que livre la genèse d'entendement est une pure matière notionnelle, un concept.

${ }^{8}$ Par exemple, avoir comme auxiliaire a un sens mais n'est pas un concept. Cela présuppose bien sûr une définition du concept comme entité sémantique «délimitée » (donc discernable), qui pourrait être discutée. Au plan syntaxique, le non-concept issu de ce type de subduction se caractérise par son absence d'autonomie : il est «non-prédicatif » (voir p. ex. Guillaume

${ }^{9}$ Théorie particulièrement développée dans les derniers écrits de Guillaume.

${ }^{10}$ Où, bien sûr, « $\mathrm{U} »$ signifie « Universel » et « $\mathrm{S} » \ll$ Singulier ».
} 
ce par quoi elles produisent, en tension I, des idées de plus en plus particularisantes, que la tension II universalise, verse à l'universel sous les traits d'une partie du discours, cas d'universalisation. (Guillaume 1982 [1957] : 63) ${ }^{11}$.

Il n'est guère nécessaire de pousser l'analyse plus avant pour conclure à la synonymie des termes «singulier» et «particulier» dans le traitement que propose Guillaume de la théorie du mot. Or cette conclusion peut être étendue à tous les systèmes où intervient l'opposition «Universel»/ «Singulier» ou «Général»/ «Particulier» ${ }^{12}$. Ce premier point étant admis ${ }^{13}$, il reste maintenant à établir avec précision ce que recouvre la notion de singularité dans le propos de Guillaume.

\section{LES SINGULIERS DU SEMANTÈME}

L'examen de la théorie de la sémantèse permet de dégager de nouvelles significations du singulier guillaumien.

\subsection{Le rôle du singulier dans la distinction entre sémantèmes et morphèmes}

Commençons par la distinction entre morphèmes et sémantèmes, définie comme le produit d'un mécanisme bien distinct de celui de l'extension nominale :

Ce mécanisme est celui par lequel un vocable, quel qu'il soit, quelles que soient son extension et sa compréhension extérieures, se replie, s'étrécit intérieurement sur un cas particulier de lui-même, plus étroit par conséquent que sa définition initiale, ou bien s'interdit à lui-même tout mouvement intérieur dans cette direction du plus particulier et du plus étroit, et ne se permet que le mouvement en sens inverse en direction du moins particulier et du plus large. (Guillaume 1991 [1945] : 149)

L'orientation vers le particulier ${ }^{14}$ qui caractérise le sémantème par opposition au morphème est de l'ordre du potentiel : elle vise à caractériser la possibilité qu'a le sémantème de s'appliquer à un cas particulier, possibilité refusée au morphème.

\subsection{L'extension singulière du substantif}

Quant au singulier de l'extension nominale ${ }^{15}$, son contenu est précisé dans diverses descriptions du système du substantif. Dans une Leçon de novembre 1943, une analyse

\footnotetext{
${ }^{11}$ Nous soulignons.

${ }^{12}$ C'est un point que je peux établir ici dans le détail, en raison du volume de citations qu'il requiert. Je signalerai en notes, au fil du texte, les variations singulier-particulier. Par convention, j'utiliserai, dans la suite de cet article, le terme « singulier » sans autre précision pour désigner indifféremment le singulier et le particulier, et le terme « particulier » seulement quand il apparaît dans les textes de Guillaume.

13 Je signale tout de même un cas très marginal chez Guillaume de distinction explicite entre singulier et particulier, figurant dans un schéma qui illustre la construction de la matière notionnelle du mot chinois, où Guillaume oppose « singulier », «idée particulière » (ou petite généralisation) et "idée générale » (ou « grande généralisation »). Voir Guillaume, 1990 [1943] : 20. Ce cas ne change rien à l'argumentation développée dans cet article.

${ }^{14}$ Le terme singulier est également utilisé à propos de la construction des sémantèmes, notamment dans Guillaume, 1991 [1945] : 125-135, 137-147.

${ }^{15}$ Guillaume utilise le plus souvent le terme «singulier» pour désigner le pôle minimal de l'extension nominale, mais parfois aussi le terme «particulier » (notamment dans Guillaume 1982 [1957] : 73-83).
} 
du concept d'extension donne d'ailleurs lieu à un intéressant développement sur la définition du singulier et de l'universel :

\begin{abstract}
Quelques-uns de mes auditeurs se demanderont peut-être ce qu'il faut entendre au juste par singulier et universel, termes déclarés ici représenter les limites infranchissables de la pensée humaine. Ces deux termes s'entendent clairement dans la philosophie de l'école. Mais il se pourrait qu'ils n'eussent pas là toute la plénitude de sens qu'ils doivent avoir dans une théorie linguistique. Voici donc des précisions, et un essai de définition appropriée étroitement à la matière linguistique.

Le meilleur discriminant en l'espèce est la notion de différence. L'universel, c'est ce qui permet à la différence un jeu illimité. Le mot chose, par exemple, est proche de l'universel parce qu'il intériorise une permission de différence très large. Le mot animal est lui aussi assez proche de l'universel parce qu'il intériorise une large permission de différence, une permission de différence plus large que celle qu'autoriserait, par exemple, le mot chien. [...]

La même définition du singulier : une interdiction de différence n'intériorisant aucune permission de différence vaut pour nos langues [...] (Guillaume, 1990 [1943] : 28)
\end{abstract}

Cette description de l'extension nominale en termes de variation de la «permission de différence » mérite quelques précisions. Elle infléchit très légèrement la conception classique de l'extension qu'ont développée les logiciens de Port-Royal - sans remettre en cause bien sûr, la loi de variation inverse de l'extension et de la compréhension. L'étendue de l'idée, selon Port-Royal, est mesurée par l'ensemble «[d]es sujets à qui cette idée convient » (Arnaud et Nicole, 1992[1662] : I, VI). Par "étendue de l'idée », c'est l'étendue du concept qui est désignée, de sorte que cette étendue est apte à se réduire jusqu'à la singularité, non seulement dans le cas du nom propre, mais aussi dans le cas où le nom subit des «additions » déterminatives conduisant à une restriction maximale $^{16}$. Mais pour Guillaume, qui est linguiste avant d'être logicien ${ }^{17}$, l'extension décrite en termes de «permission de différence » est une propriété du mot - en l'occurrence ici du substantif -, pas du concept, et une propriété qui ne caractérise, comme on l'a vu plus haut, que l'aspect extérieur du mot. De sorte que la restriction du côté du singulier est limitée, pour tout sémantème, en amont de la singularité du nom propre. Au-delà de cette limite, le mot n'est plus un sémantème mais un « asémantème » : l'équilibre de la différence interdite et de la différence permise est en effet rompu dans le cas du morphème mais aussi lorsque

\begin{abstract}
la permission de différence s'est éteinte sous la pression même de l'interdiction de différence devenue de plus en plus étroite et stricte. Il en est ainsi quand se substitue au nom commun le nom propre. Le nom propre est un asémantème et il a pour déterminant le renforcement de l'interdiction de différence jusqu'à un degré où la différenciation intérieure est une impossibilité. C'est ainsi que si je désigne une personne par son nom propre, ce nom pose une interdiction de différence, une interdiction d'en faire le nom d'une autre personne, laquelle interdiction ne souffre intérieurement d'être contrariée par aucune impulsion en sens contraire. Un nom propre, parce qu'au-dedans de l'interdiction de différence qu'il pose il ne fait aucune place à la permission de différence, est asémantique. (Guillaume 1991 [1945] : 118)
\end{abstract}

En outre, mais c'est un point que je ne développerai pas ici, le concept de différence introduit par Guillaume pour définir l'extension est marqué par l'héritage saussurien de

\footnotetext{
16 «Et ces additions sont quelquefois telles, qu'elles rendent individuel un mot général, quand on y ajoute des conditions individuelles, comme quand je dis : Le pape qui est aujourd'hui, cela détermine le mot général de pape à la personne unique et singulière d'Alexandre VII » (Arnaud et Nicole, 1992[1662] : I, VIII). Ajoutons que la logique de Port-Royal considère que ce genre de détermination peut ne pas être exprimée mais fournie par le contexte («comme quand nous disons en France le roi » (ibid.))

${ }^{17} \mathrm{Ou}$ si l'on préfère logicien pour autant qu'il est linguiste.
} 
la théorie de la valeur: Guillaume utilise la même expression de «permission de différence » pour caractériser le phonème dans son essence différentielle ${ }^{18}$. Par conséquent, la définition de l'extension du sémantème en termes de permission et d'interdiction de différence permet à la fois de caractériser la nature différentielle du sémantème et d'expliquer le mécanisme de variation d'extension.

Au total, il convient donc de distinguer, dans le cas du sémantème, trois types de singularités : la singularité du sémantème en général (par opposition au morphème), la singularité du nom commun (qui préserve une «permission de différence », aussi minime qu'elle soit) et la singularité du nom propre, qui se définit en termes strictement extensionnels, par le fait qu'il ne peut s'appliquer qu'à un seul référent ${ }^{19}$, ces trois singularités s'ajoutant à celle du mot non subduit, examinée dans la première section de cet article.

\section{SINGULIER NUMÉRIQUE ET SINGULIER ANUMÉRIQUE}

Mais il faut encore compter avec un autre type de singularité, peut-être le plus évident, qui est impliqué dans les systèmes du nombre et de l'article.

\subsection{Articles et singularité}

Je passerai rapidement sur le célèbre système de l'article, en rappelant tout de même, pour mémoire, qu'il est décrit selon un schéma du type suivant (Guillaume, $1991[1945]: 208)^{20}:$

\footnotetext{
18 «a prononciation effective se compose d'une multitude infinie de différences, ramenées par psychisation, par une psychisation généralisante, à un nombre restreint de conditions permissives idéelles. Un - $r$-, par exemple, est l'indication d'une prononciation permissive à l'égard de différences assujetties seulement, si nombreuses soient-elles, à ne pas sortir de la permission accordée. »(Guillaume, 1973 [1948] : 38)

${ }^{19}$ L'analyse du nom propre par Guillaume mériterait sans doute un plus ample développement. Je relèverai seulement qu'en définissant le nom propre comme asémantème Guillaume se garde bien de le définir comme vide de sens. L'asémantème a un sens, mais d'une qualité ou d'une nature distincte de celui du sémantème.

${ }^{20}$ On notera que ce schéma utilise simultanément les notions de singularité et de particularité.
} 
Or, comme on peut le constater sur ce schéma, ce singulier ${ }^{21}$ se dédouble en raison de sa position intermédiaire. Guillaume distingue en effet le singulier comme terme final de la pluralité interne (I/1) du singulier comme terme initial de la pluralité externe $(1 / \mathrm{I})^{22}$. On obtient donc, finalement, quatre singuliers distincts: le singulier de l'article un (particularisation maximale), le singulier de l'article le (généralisation minimale), le singulier de la fin de la pluralité interne et le singulier du début de la pluralité externe, que Guillaume assimile à la singularité numérique, au nombre $1^{23}$.

Cependant, la simple juxtaposition des «psychomécanismes » du nombre et de l'article ne rend pas compte de la filiation historique de ces systèmes, et plus précisément du fait que, selon Guillaume, les langues à articles ont élaboré ce système continu anumérique à partir du système du nombre, en vidant ce dernier de sa substance quantitative discontinue pour ne retenir que les tensions de l'universel au singulier et du singulier à l'universel ${ }^{24}$. De sorte que les quatre singuliers qui viennent d'être identifiés ne coexistent que logiquement, ou si l'on préfère dans une vision synoptique de l'ensemble des solutions possibles pour toute langue, mais jamais dans une langue particulière. C'est ce qui apparaît bien dans la Leçon du 23 avril 1959, qui distingue clairement le singulier de l'article et le singulier du nombre :

En figure explicative, $S_{a}$ notant le singulier numérique et $S_{z}$ le singulier anumérique qualitatif, le singulier d'originalité, un système se développe formé de deux plans dont le tout est figurativement ce que je porte de nouveau au tableau :

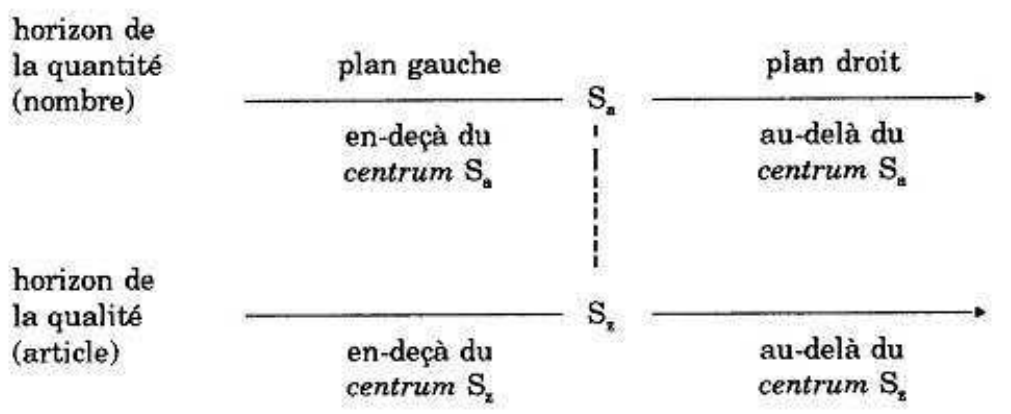

\footnotetext{
${ }^{21}$ Dans le cas du système du nombre, Guillaume n'utilise jamais le terme «particulier » mais toujours le terme «singulier», ce qui s'explique, comme on l'a vu, par le rôle central du «singulier numérique » dans ce système.

${ }^{22}$ «Un fait secret très important est que la formation de singulier, devenue, après élimination du pluriel interne, le domaine du continu, ne saurait quitter la catégorie du nombre avant qu'ait été inventé un singulier appartenant exclusivement à la formation du pluriel. L'invention de ce singulier est une chose accomplie à partir du moment où il est fait analytiquement la distinction de deux singuliers limites : celui I/1 qui termine la formation de singulier et lui appartient encore, et celui inversé 1/I qui ouvre la formation de pluriel et lui appartient déjà. » (Guillaume, 1991 [1945] : 216)

${ }^{23}$ Cette conception du système du nombre prête le flanc à diverses critiques. On peut notamment contester la symétrie du système : même dans les langues où un pluriel interne existe, le pluriel externe présente une structure incomparable au pluriel interne (voir notamment Serbat, 1993). Il est par ailleurs envisageable d'intégrer au pluriel interne les déterminants indéfinis complexe du type «une foule de », etc. (Serbat, 1993, Moignet, 1981). On peut enfin défendre l'idée que le point initial de la série arithmétique se situe au voisinage de zéro, pas de 1.

${ }^{24}$ Plus précisément, dans une langue à articles (comme le français), la tension I du système du nombre, qui porte le pluriel interne, a disparu (voir notamment Guillaume, 1985 [1946] : 195). On rappellera également que l'analyse du système du nombre par Guillaume s'inscrit dans une tentative d'expliquer la disparition du pluriel interne en français, conçu comme une sorte d'aberration structurelle et donc condamné à l'élimination.
} 
Le diagramme porté au tableau enclôt les deux théories parallèles du nombre et de l'article et permet, attentivement considéré, de résoudre tous les problèmes posés différemment et résolus différemment, suivant les idiomes, sur les deux horizons, l'horizon haut de la quantité et l'horizon bas de la qualité, dans le plan gauche du système construit, puis dans le plan droit. (Guillaume, 1995 [1959]:189)

On notera d'ailleurs que cette idée selon laquelle le singulier de l'article ne se confond pas avec le singulier numérique est constante chez Guillaume. Voici comment il la formule dans une Leçon du 16 mai $1947^{25}$ :

Autre chose $^{26}$ est de dire : J'ai une femme. Dans ce dernier exemple, le mot une est un article, allant vers l'idée de singulier numérique, qu'il n'atteint pas, et que l'esprit situe à quelque distance, dans la perspective directionnelle. Soit figurativement, en linguistique de position :

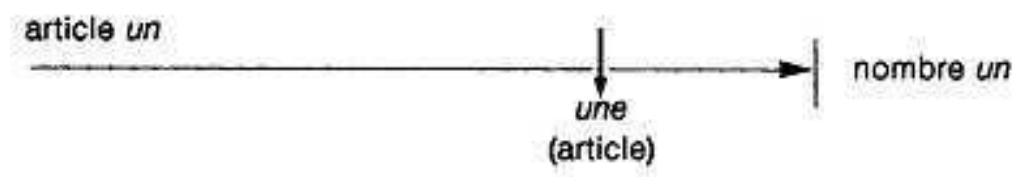

(Guillaume (1989) [1947] : 183 ; nous soulignons)

Le système de l'article est donc bien une «similitude » du système du nombre, mais les deux systèmes restent distincts, ainsi que les singularités qu'ils produisent.

\section{LE SINGULIER AU-DELÀ DES SYSTÉMES}

Si l'approche guillaumienne du singulier dans les systèmes de la langue est d'une richesse remarquable, c'est que les tensions que le linguiste met au jour dans son analyse des faits de langue proviennent d'une propriété fondamentale de la cognition humaine $^{27}$ :

Ces prémisses posées $[\ldots]$ nous avons à revenir au fait qui domine, en tout temps et en toute époque, la structure d'une langue quelle qu'elle soit, à savoir que tout ce que la pensée entreprend de constructif a lieu entre des limites qui marquent les bornes de l'esprit et qui sont d'un côté le singulier et de l'autre l'universel. L'intervalle inscrit entre ces limites, que la pensée, quel que soit son effort pour le faire, ne saurait franchir, est ce que nous nommerons le diastème psychique fondamental.

Au sein de ce diastème, le mouvement de la pensée peut être un mouvement de généralisation se propageant à partir du singulier en direction de l'universel, ou bien un mouvement de

\footnotetext{
${ }^{25}$ Cette distinction est présente dès Le problème de l'article (Guillaume, 1975 [1919]) où Guillaume ne traite que les emplois «impressifs » de un qu'il oppose à son emploi «expressifs », c'est-à-dire numéral (voir Guillaume 1919: )

${ }^{26}$ Guillaume oppose ici «J'ai une femme» à «J'en ai une de femme». Je ne commenterai pas la bizarrerie de ces phrases dans le contexte d'une civilisation monogame...

${ }^{27}$ Cette propriété est elle-même issue du rapport univers/homme : «L'édification des langues repose sur le rapport permanent, inévitable (et dans l'en deçà duquel on n'en découvre pas d'autre) univers/homme le rapport univers/homme étant la racine concrète du rapport universel/singulier, partout présent et opérant en glossogénie, présent au point que rien ne peut être expliqué de ce qu'est, par structure et par architecture, le langage humain sans partir, pour l'expliquer et fructueusement l'observer, dudit rapport » (Guillaume, 1995 [1959] : 140).
} 
particularisation se propageant en direction du singulier à partir de l'universel. (Guillaume 1991 [1945] : 126$)^{28}$

Il n'est donc pas étonnant que la polarité de l'universel et du singulier soit impliquée dans l'acte de langage lui-même, c'est-à-dire dans la transition langue-discours.

\subsection{La singularité discursive de l'actualisation}

Selon l'expression de Louis Havet, Gustave Guillaume est un linguiste psychologue ${ }^{29}$ : il s'intéresse essentiellement aux opérations mentales qui sont à l'origine des systèmes situés au niveau de la langue. Le niveau du discours s'oppose, comme plan de l'effet, au niveau de langue comme plan de la puissance. Or cette transition du puissanciel à l'effectif est aussi un accès à une forme de singularité :

«Le discours se développe sur le plan de l'effet, dans le moment où la parole est jugée utile par celui qui en fait usage. Il a pour attribut la momentanéité et la singularité. »(Guillaume 1985 [1945]: 35)

Cette singularité, celle de l'actualisation ${ }^{30}$, correspond à l'effectivité d'un acte cognitif, qui aboutit à la production d'une phrase, unité d'effet du discours. Il s'agit donc, dans le vocabulaire de la philosophie du langage contemporaine, d'un fait d'indexicalité mentale $^{31}$, c'est-à-dire de l'occurrence d'une pensée dans un esprit, mais ici d'une pensée indissociable d'un exprimé discursif. Cependant cette singularité de nature mentale en entraîne d'autres dans son sillage :

Les choses que mon discours énonce sont des choses momentanément conçues et qui, momentanément, s'adressent à un interlocuteur sur lequel le sujet parlant entend agir d'une manière singulière dans le moment même où il parle. L'action que le discours tend à exercer sur l'interlocuteur est singulière, en ce sens qu'elle se rapporte à un cas de contact original qui n'est pas censé devoir se répéter. Le sujet parlant a devant lui, comme objectif, autant d'actions singulières exercées sur l'auditeur qu'il est sujet à en concevoir, à en imaginer, à en supposer d'utiles. Ce domaine, parce qu'il est celui du singulier infiniment renouvelable, ne connaît pas théoriquement de limitation. La parole humaine développe à l'infini sa capacité d'énoncer des choses singulières, non encore dites et non sujettes à répétition identique. (ibid.)

Il ne s'agit plus ici de la singularité de l'acte cognitif du dire, mais de ce qui en résulte, la singularité de principe du dit, des phrases effectivement produites. On notera au passage que Guillaume ne manque pas d'envisager le singulier de la dimension perlocutoire du discours.

\subsection{Singularité et référence}

\footnotetext{
${ }^{28}$ On notera que le singulier et le particulier sont mêlés dans cette définition du «diastème psychique fondamental ».

${ }^{29}$ Voir l'extrait du Journal des savants publié en ouverture du Problème de l'article (Guillaume, 1975 [1919] : XV).

${ }^{30}$ Je laisse ici de côté le problème posé par la question de l'actualisation chez Guillaume si l'on accepte la distinction entre «effet de sens » et «signifié d'effet». Car dans ce cas, on ne sait pas si le processus d'actualisation s'achève avec l'effet de sens ou avec le signifié d'effet. Il serait possible de montrer que l'effet de sens représente, par rapport au signifié d'effet, un pas de plus en direction du singulier, et par conséquent une actualisation plus développée.

${ }^{31}$ Sur la notion d'indexicalité mentale, voir par exemple Récanati, 2008 : 192-208.
} 
Ce rapide parcours des singularités guillaumiennes aura permis, je l'espère, de donner un aperçu de l'extraordinaire finesse qui caractérise l'approche guillaumienne du singulier. De l'aptitude cognitive générale à osciller entre l'universel et le singulier, découlent les multiples singularités impliquées par les systèmes de la langue ainsi que la singularité de l'actualisation comme fait d'indexicalité mentale et la singularité du discours effectif.

Il y manque cependant un type de singularité, qui est sans doute celui qui a suscité l'investigation philosophique la plus intense : la singularité du référent, des êtres et des choses du monde. De Scott ou Ockham à Strawson, en passant par Leibniz et de nombreux autres, la question du singulier est en effet liée à celle du réel ou de l'existant. La thèse la plus radicale à cet égard peut être formulée de la façon suivante : tout ce qui est réel est singulier; tout ce qui est singulier est réel ${ }^{32}$. Il ne m'appartient pas ici de justifier, d'argumenter ni de développer cette thèse. Je la mentionne parce qu'elle permet de mettre en évidence une insuffisance majeure des théories linguistiques de la référence $^{33}$ : certes, le linguiste n'est pas philosophe, mais comment peut-il prétendre thématiser la relation du langage au réel en limitant son interrogation à la sphère linguistique, c'est-à-dire sans jamais questionner le sens ou la nature du réel lui-même ? La réponse apportée par la thèse singulariste permet donc de poser les bases d'une approche linguistique de la référence fondée sur une conception explicite du réel ${ }^{34}$. Or, si l'on admet que le réel est composé de singularités, le problème de la référence devient celui de la rencontre entre les singularités du monde et les singularités linguistiques. L'apport guillaumien pourrait donc être développé en direction d'une théorie singulariste de la référence. En particulier, l'idée d'un singulier polaire, c'est-à-dire visé sans jamais être atteint, suggère assez bien la complexité du rapport que l'homme entretient avec le réel - un réel qui n'est entrevu qu'à la faveur d'une expérience spéciale $^{35}$, et parfois tragiquement évité ${ }^{36}$. Le singulier serait alors ce à quoi l'on ne

\footnotetext{
32 On nommera «thèse singulariste » toute conception du réel qui accepte cette formulation (quelle que soit la façon dont le concept de singularité est défini).

${ }^{33}$ J'entends par théorie de la référence une théorie qui s'efforce d'expliquer ou de modéliser la relation du langage au monde réel, « extralinguistique ».

${ }^{34}$ On peut distinguer, outre la théorie fondée sur la thèse singulariste, trois grandes familles de théories de la référence : les théories éliminativistes, pour lesquelles la question de la référence n'est pas pertinente, parmi lesquelles il est possible d'effectuer une distinction, fondée sur la raison alléguée de cette nonpertinence, entre l'éliminativisme structuraliste (raison d'ordre épistémologique) et l'éliminativisme psycho-social (pour qui la référence ne concerne pas le monde mais seulement les relations entre les membres d'une communauté linguistique) ; les théories empiristes, qui admettent sans l'interroger ce que Merleau-Ponty nomme la «thèse du monde », c'est-à-dire qui présupposent, conformément à l'attitude naturelle, l'existence d'un monde réel accessible par les sens et apte à être désigné de façon transparente par les mots du langage; les théories phénoménologiques, qui suspendent la thèse du monde, donc le face-à-face résultatif langage-monde, pour privilégier l'analyse de la constitution du rapport entre le langage et le monde tel qu'il apparaît à l'homme, comme phénomène.

${ }^{35}$ L'expérience ordinaire ou l'attitude naturelle ne livrent généralement que des particuliers, pas des singuliers; des particuliers au sens d'éléments de catégories ou d'occurrences de généralités (p. ex. une ville comme élément de l'ensemble des villes), qui s'opposent au singulier comme incomparable, irréductible à un membre de catégorie (p. ex. cette ville précise, dans laquelle je vis, et que je perçois parfois dans sa singularité, comme absolument incomparable). Le célèbre aphorisme de Quine, selon lequel «être, c'est être la valeur d'une variable liée », ne vaudrait donc dans cette perspective que pour l'existence des particuliers.
} 
cesse de faire référence; il serait l'irréféré, tout comme l'intraduisible, selon l'heureuse formule de Barbara Cassin, est ce que l'on ne cesse de traduire ${ }^{37}$.

Philippe Monneret Université de Bourgogne

\footnotetext{
${ }^{36}$ Tel est le point de vue de Clément Rosset, qui montre comment le tragique naît de la construction par l'homme de doubles du réel, ces doubles permettant d'éviter le réel lui même (voir Rosset (1976) et Rosset (1979)).

${ }^{37}$ «L'intraduisible, c'est plutôt ce qu'on ne cesse pas de (ne pas) traduire » (Cassin, 2004 : XVII).
} 